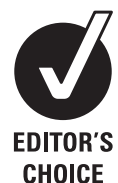

Associate Editor, Evidence Based Medicine, Department of Family Medicine, University of Alberta, Canada

Correspondence to:

Denise Campbell-Scherer

Associate Professor,

Department of Family Medicine,

University of Alberta,

Grey Nuns Family

Medicine Centre,

2927-66 St Cedars Professional

Park, Edmonton,

Alberta, T6K 4C1,

Canada;

denise.campbell-scherer@

ulberta.ca

\title{
Multimorbidity: a challenge for evidence-based medicine
}

\section{Denise Campbell-Scherer}

In keeping with the challenge presented by our Editor, of how to translate evidence into policy and practice, ${ }^{1}$ this article will focus on a fundamental barrier: 'multimorbidity'.

Multimorbidity refers to the co-occurrence of two or more chronic conditions in one patient. ${ }^{2}$ Management targeting one condition in a patient may cause undesirable sequelae with regard to their other conditions. Some examples include non-steroidal anti-inflammatory medications for pain relief from arthritis, which aggravate hypertension and renal disease, diuretic medications for heart failure causing exacerbation of renal failure, aspirin for heart disease with the potential of causing bleeding in patients with gastric ulcers and steroids for inflammatory and autoimmune conditions causing high glucose levels in diabetics. Yet patients with multiple chronic diseases are often excluded from clinical trials that constitute the bulk of the evidence supporting treatment for specific conditions. $^{3}$ This exclusion might not be a substantial concern if multimorbidity was rare, but it is not.

In primary care, $45 \%$ of patients have multimorbidity. In older adults this increases to $50 \%$ of those older than 65 years having three or more co-morbid conditions and 20\% having five or more conditions. The prevalence is increasing, with more than $50 \%$ of the US population expected to have a chronic disease by the year 2020 . [4, 5 as cited in 6] These patients tend to have increased disability, depression, anxiety and rapid declines in health status. ${ }^{6}$ Currently, 75\% of healthcare expenditures in the USA are for chronic conditions. ${ }^{4}$ Also in the USA, for those covered by Medicare (government health insurance for patients aged 65 and older, and people of all ages with renal transplant or on dialysis, or who are disabled), 80\% of expenses are devoted to patients with four or more chronic conditions. Costs increase exponentially with the number of chronic conditions. ${ }^{6}$

Multimorbidity is common and costly, yet we have little evidence for efficacy of treatments for diseases in such patients. Policy makers and clinicians look to rigorously derived, evidence-based clinical practice guidelines (CPGs) to improve quality of care, and allocate scarce resources logically. The exclusion of patients with multimorbidity from efficacy studies, however, makes applicability of CPGs questionable. Most current CPGs focus on a single disease or condition. Few CPGs address the reality that patients present with multiple chronic conditions simultaneously. As a result, determining standards for clinical practice based upon CPGs is fraught with hazard.

In addition to a number of threats to the validity of evidence and misleading reporting of study results that have been well described in the literature, ${ }^{7-11}$ there are significant problems with generalisability when the patients in the 'real world' with multimorbidity are excluded from clinical trials. Trial results may also not apply to the elderly for similar reasons. Scott and Guyatt ${ }^{12}$ have summarised decision steps, barriers and potential solutions to the application of evidence-based medicine (EBM) in older patients, who often have significant multimorbidity. Fortin and colleagues ${ }^{3}$ have evaluated multimorbidity in the inclusion and exclusion criteria of randomised controlled trials (RCTs). Their study demonstrates that RCTs targeting a chronic medical condition such as hypertension could find that most eligible patients from a family practice would have comorbid conditions. They found that the studies underpinning guideline recommendations excluded patients with some comorbid conditions, and did not comment on patients with multiple comorbidities. The concern is that for results to be generalisable to practice, they need to reflect the conditions in the population.

Unfortunately, lower quality evidence underpins many guideline recommendations now, even aside from the issue of generalisability. ${ }^{13}$ Schema can help physicians, patients and policy makers identify strong versus weak $\mathrm{CPG}$ and recommendations. One excellent example is the use of the GRADE system (Grading of Recommendations Assessment, Development, and Evaluation). ${ }^{14}$

There is a disconnect between healthcare focusing on the individual patient versus the individual disease. Boyd and colleagues ${ }^{15}$ have highlighted the conflict in their reflections on the implications of pay for performance in patients with multimorbidity, stating that "standards that define quality of primary care by placing emphasis on high rates of adherence to guidelines and targets rather than weighing the burden, risks and benefits of complex therapies in shared decision making could ultimately undermine the quality of care."

Given the overuse (and misuse) of the term evidencebased medicine (EBM), it is worthwhile to review the definition. "EBM is the conscientious, explicit and judicious use of current best evidence in making decisions about the care of individual patients. The practice of EBM requires integration of individual clinical expertise and patient preferences with the best available external clinical evidence from systematic research". ${ }^{16}$ It is clear, and has been well known for some time, that EBM does not mean simply doing what has been proven to have efficacy in clinical trials and systematic reviews.

Current evidence for treatment of a given condition requires interpretation within the context of a patient's health and situation, in order to safely and judiciously apply it. This is particularly true in patients with multimorbidity where the potential for medication interactions, unwanted or unanticipated effects or contraindications to interventions are greatly increased. Given the prevalence of these complex patients in primary care particularly, caution must be used in designing clinical decision support systems, applying guidelines and in deciding on implementation of task-based quality metrics. Guidelines are excellent tools, but bad masters in the improvement 
of quality in primary care. With the need to extrapolate existing evidence to individual patient situations, the most valid approach is to embrace evidence-based patient choice, also referred to as shared decision making. ${ }^{17}$

In the absence of evidence in patients with multimorbidity, what can clinicians and researchers do? Braithwaite and colleagues have proposed one strategy for tailoring clinical guidelines to comorbidity at the point of care. They propose using a payoff time calculation. They define this as the minimum elapsed time until the cumulative incremental benefits of the intervention exceed their cumulative incremental harms, weighed against the patient's comorbidity-adjusted life expectancy. ${ }^{18}$ While these strategies may be useful for some recommendations like screening tests, they are less useful for individual treatment decisions. For these, clinicians need to prioritise conditions based upon severity and patient importance. For researchers, it is important to specify the characteristics of patients recruited to studies, and to recruit broadly with regard to multimorbidity and age. In the interim, there is a possibility of doing subgroup meta-analyses; however, given the limitations of subgroup analyses, it is important to do this carefully and to be circumspect in the reporting of results. ${ }^{19}$

Multimorbidity represents the next frontier in the evolution of EBM. It is necessary to begin doing more research on chronic diseases and multimorbidity in primary care populations. Research in the area will not be easy - but to quote Dr. Kerr White, ${ }^{20}$ "All really important research is difficult. If it is not difficult it might not be worth doing. Others have tackled the easy problems."

Competing interests: I do not have any financial competing interests. Professionally I am doing research related to multi-morbidity and as a primary care physician I care for patients with multi-morbidity.

\section{References}

1. Saitz R. Evidence-based medicine: time for transition and translation (to practice). Evid Based Med 2010;15:103-4.

2. Akker M vd, Buntinx F, Knottnerus A. Comorbidity or multimorbidity: what's in a name? A review of literature. Eur J Gen Pract 1996;2:65-70.

3. Fortin M, Dionne J, Pinho G, et al. Randomized controlled trials: do they have external validity for patients with multiple comorbidities? Ann Fam Med 2006;4:104-8.
4. Hoffman C, Rice D, Sung HY. Persons with chronic conditions. Their prevalence and costs. JAMA 1996;276:1473-9.

5. Wu SY, Green A. Projection of Chronic Illness Prevalence and Cost Inflation. Washington, DC: RAND Health 2000.

6. Wolff JL, Starfield B, Anderson G. Prevalence, expenditures, and complications of multiple chronic conditions in the elderly. Arch Intern Med 2002;162:2269-76.

7. Chan AW, Hróbjartsson A, Haahr MT, et al. Empirical evidence for selective reporting of outcomes in randomized trials: comparison of protocols to published articles. JAMA 2004;291:2457-65.

8. Bero L, Oostvogel F, Bacchetti P, et al. Factors associated with findings of published trials of drug-drug comparisons: why some statins appear more efficacious than others. PLoS Med 2007;4:e184.

9. Buchkowsky SS, Jewesson PJ. Industry sponsorship and authorship of clinical trials over 20 years. Ann Pharmacother 2004;38:579-85.

10. Bassler D, Montori VM, Briel M, et al. Early stopping of randomized clinical trials for overt efficacy is problematic. J Clin Epidemiol 2008;61:241-6.

11. Montori VM, Jaeschke R, Schünemann HJ, et al. Users' guide to detecting misleading claims in clinical research reports. $B M J$ 2004;329:1093-6.

12. Scott IA, Guyatt GH. Cautionary tales in the interpretation of clinical studies involving older persons. Arch Intern Med 2010;170:587-95.

13. Tricoci P, Allen JM, Kramer JM, et al. Scientific evidence underlying the ACC/AHA clinical practice guidelines. JAMA 2009;301:831-41.

14. Guyatt GH, Oxman AD, Vist GE, et al.; GRADE Working Group. GRADE: an emerging consensus on rating quality of evidence and strength of recommendations. BMJ 2008;336:924-6.

15. Boyd CM, Darer J, Boult C, et al. Clinical practice guidelines and quality of care for older patients with multiple comorbid diseases: implications for pay for performance. JAMA 2005;294:716-24

16. Sackett DL, Rosenberg WM, Gray JA, et al. Evidence based medicine: what it is and what it isn't. BMJ 1996;312:71-2.

17. Edward A, Elwyn G, eds. Shared Decision-Making in Health Care. Achieving Evidence-Based Patient Choice. Second edition. Oxford UK, Oxford University Press 2009: 3-10.

18. Braithwaite RS, Concato J, Chang CC, et al. A framework for tailoring clinical guidelines to comorbidity at the point of care. Arch Intern Med 2007;167:2361-5.

19. Sun X, Briel M, Walter SD, et al. Is a subgroup effect believable? Updating criteria to evaluate the credibility of subgroup analyses. BMJ 2010;340:c117.

20. White KL. Fundamental research at primary care level. Lancet 2000;355:1904-6. 